\title{
Silver Nanoparticles Impregnated Biocellulose Produced by Sweet Glutinous Rice Fermentation with the Genus Acetobacter
}

\author{
Duongruitai Nicomrat ${ }^{1, *}$ \\ ${ }^{1}$ Division of Environmental Sciences and Natural Resources, Faculty of Science and Technology, Rajamangala University of \\ Technology Phra Nakhon, Bangkok, Thailand
}

\begin{abstract}
Bacterial cellulose $(\mathrm{BC})$ is a natural renewable and biodegradable polymer and mostly synthesized from the bacteria genus, Acetobacter. More recent studies on effective biopolymer in various applications have been conducted due to its uniform structure and morphology superior to those of plant cellulose. In this study, the researchers have observed the effectiveness on antimicrobial activity of BC produced by sweet glutinous rice as a raw material instead of cellulosic materials. In the process, sweet glutinous rice fermented by Loog-pang was fermented for alcohol before further making rice vinegar product by inoculating with Gluconacetobacter xylinum TISTR 086, or mixed with indigenous vinegar producers, isolated Acetobacter spp. from our laboratory. Both these two samples gave comparable BC yields and the antimicrobial activity after impregnating with silver nanoparticles and also comparable to the control coconut vinegar medium (2.20 vs $2.00 \mathrm{~g} / \mathrm{L}$ within 3 days). The optimum conditions for high $\mathrm{BC}$ production were $\mathrm{pH} 4$ to 7 , temperature approximately $25-30^{\circ} \mathrm{C}$, slow agitation (50 to $90 \mathrm{rpm}$ ) as well as static cultivation. The remarkable antimicrobial activities of silver nanoparticles impregnated $\mathrm{BC}$ products (Ag-BC) showed high tentative in vitro inhibition on common pathogens in contaminated wounds; Staphylococcus aureus, Escherichia coli and Pseudomonas aeruginosa by agar diffusion test. From this study, Ag-BC produced by fermented sweet glutinous rice (FSR) will therefore be one attractive affordable choice for being biomaterials applied in medical interests, especially skin disinfectant.
\end{abstract}

Keywords. Bacterial cellulose; silver; mixed Acetobacter; glutinous rice vinegar

\section{Introduction}

Biocellulose from bacteria or bacterial cellulose (BC) is a natural biopolymer mainly produced by the bacterium Gluconacetobacter xylinus [1]. BC with a three-dimensional network structure is composed of microfibrils that have nanometric diameters and has intensively been studied over the past ten years due to its remarkable and unique physicochemical, mechanical, and biological properties $[2,3]$. Since $\mathrm{BC}$ membranes present relatively high porosity allowing the incorporation or synthesis in situ of silica $\left(\mathrm{SiO}_{2}\right)$ or metallic nanoparticles [4]. Moreover, BC have been applied in biomedicine area, such as biomaterials in tissue engineering, scaffolds, wound dressing, food packaging, drug release [2, 5-7].

Many researches on bioactive $\mathrm{BC}$ material have recently introduced to food technology as antimicrobial packaging, active packaging that extends shelf-life and food safety of various products $[8,9]$. The initial application of BC by Fontana et al. (1990) [10] was the BC product made from fermented coconut juice vinegar as cellulose dressings, temporary covering for the treatment of wounds. It could be applied as wet BC in the treatment of partial thickness burns $[11,12]$. It promotes a favourable moist environment for a fast wound cleansing, and consequently for rapid healing. In addition, their work indicated that the $\mathrm{BC}$ wound dressing could shorten the time to heal or wound closure over standard care. Recent emerging biomaterials produced by $\mathrm{BC}$ membranes were being used in small diameter blood vessel replacement [12] and as temporary skin substitute in the treatment of chronic wounds, burns, and ulcers [4, 10].

Since silver nanoparticles is similar to that of silver ion but its advantages over other antimicrobial agents are its reducing the resistance of various antibiotics. Additionally, it can be embedded or impregnated into natural biopolymers in a variety of biomedical materials and devices. Recently researches also had tested for the technique of silver nanoparticles (AgNPs) incorporation into $\mathrm{BC}$ gel as antimicrobial support extended the biomedical treatments as antibacterial wound dressing $[4,13]$. Their works based on AgNPs combined into gel or blended with a gelling agent to form a gel wound dressing. Most developed Ag-BC gel application done by diluted silver nanoparticles at a concentration from 1,000 to $10,000 \mathrm{ppm}$ in the gel of $\mathrm{BC}$ have been researched that it can prevent several pathogenic bacteria on the patients' wound. In addition, others applications of BC were to medical implants, drug delivery, vascular grafts, and scaffolds for tissue engineering etc. [14-16]. Overall works have, therefore, brought the attention to the researchers to comprehend the antibacterial activity on especially wound pathogens based on $\mathrm{Ag}-\mathrm{BC}$ from fermented sweet glutinous rice

\footnotetext{
* Corresponding author: duongruitai.n@rmutp.ac.th
} 
(FSR) compared with that from common $\mathrm{BC}$ made by coconut juice. With AgNPs impregnated BC membrane (Ag-BC) at the concentrations of 10 up to $1,000 \mathrm{ppm})$, the Ag-BC would be determined for its effectiveness in inhibiting pathogenic contaminants presented in wounds. The inhibition activity was based on in vitro disk diffusion method. Once, the advantages of $\mathrm{BC}$ membrane produced by FSR would be addressed and brought such an alternative choice of bio-wound dressing that harbours high inhibition effects on pathogens.

\section{Materials and Methods}

\subsection{BC Membrane Production}

The sample of culture medium, rice vinegar obtained by fermentation of sweet glutinous rice (FSR), which was previously cooked and washed with water several times before mixing with Loog-Pang (rice cake starter, a traditional starter culture for production of alcoholic foods and drinks in southern Thailand) (e.g. $5 \mathrm{~g} / \mathrm{L}$ and incubating for 3 to 4 days until ethanol at least $5 \%(\mathrm{v} / \mathrm{v})$ was detected [17]. This medium was diluted to obtain ethanol $2 \%(\mathrm{v} / \mathrm{v})$. Another control medium, coconut vinegar was from the fermentation supplemented with glucose $100 \mathrm{~g} / \mathrm{L}$, yeast extract $4 \mathrm{~g} / \mathrm{L}, \mathrm{Na}_{2} \mathrm{HPO}_{4} 2 \mathrm{~g} / \mathrm{L}$, $\mathrm{MgSO}_{4} \cdot \mathrm{H}_{2} \mathrm{O} 0.8 \mathrm{~g} / \mathrm{L}$, and ethanol 2\% (w/v) and fermented by Saccharomyces cerevisiae EC1118. Both media were sources of culturing media for $\mathrm{BC}$ membrane. Total bacterial counts during the fermentation were from total plate counts of serial diluted bacteria on Hestrin Schramm (HS) agar composed of glucose $2.0 \%$, peptone $0.5 \%$, yeast extract $0.5 \%, \mathrm{Na}_{2} \mathrm{HPO}_{4} 0.27 \%$, citric acid $0.15 \%$ $(\mathrm{v} / \mathrm{v})$ and agar $1.5 \%$ [18].

\subsection{The Conditions for Bacterial Cellulose Preparation}

$\mathrm{BC}$ membranes were obtained from cultivation of the Acetobacter xylinum TISTR 086 or mixed cultures of Acetobacter spp., the isolates from natural vinegar fermentation of Thai white glutinous rice obtained in our laboratory. These bacteria were incubated statically in Hestrin Schramm (HS) media at $\mathrm{pH} 6.0,30^{\circ} \mathrm{C}$ for 7 days. Culture medium types including fermented sweet glutinous rice (FSR) and coconut, the control sample, were set for bacterial culturing for 2 to 15 days. The culturing conditions were varied for the $\mathrm{pH}$ ranging at 3-7.5 and temperature at $20-35^{\circ} \mathrm{C}$ including static incubation in trays of $30 \times 50 \mathrm{~cm}$ and agitating at $90 \mathrm{rpm}$. These membranes were thus washed several times in water, $2 \%$ aqueous sodium hydroxide at $70^{\circ} \mathrm{C}$, in order to remove all color and microbial debris, and then water until neutral $\mathrm{pH}$.

\subsection{BC membranes impregnated with AgNP solution}

The BC membranes were immersed in silver nanoparticle (AgNP) solution (Prime Nanotechnology Co., LTD,
Bangkok, Thailand) at the concentration of 10, 50, 100, and $1,000 \mathrm{ppm}$ and slowly shaken for $36 \mathrm{~h}$ with frequently exchanges performed in order to ensure substitution of water for AgNP solution. The BC membranes saturated with AgNP solution (Ag-BC) was kept at $4^{\circ} \mathrm{C}$ for further use.

\subsection{Disk Diffusion Method for Antibacterial Activity}

Antibacterial Assay. The disk diffusion method (Clinical and Laboratory Standards Institute (CLSI) (CLSI, 2009) was employed in this study, with some modifications, to evaluate the antibacterial activity of the Ag-BC against Escherichia coli ATTC 25922, Staphylococcus aureus ATCC 25923, Pseudomonas aeruginosa (gifted by Siriphatr Chamutpong, Faculty at RMUTT, Bangkok, Thailand). The bacterial suspension in nutrient broth was prepared by culturing for $24 \mathrm{~h}$ and diluted until its turbidity equivalent to a 0.5 McFarland standard (approximately $10^{8} \mathrm{CFU} / \mathrm{mL}$ ). The suspension was spread onto the surface of Mueller Hinton agar contained in a plate $(90 \times 15 \mathrm{~mm})$ by cotton swab. Ag-BC Membranes cut in disks (diameter $5.5 \mathrm{~mm}$ ) were applied to the agar surface and $5 \mu \mathrm{L}$ of nutrient broth was put on the surface of each BC membrane. BC membrane without AgNPs submitted to the same procedure of the Ag-BC samples was used to control the experiment. The plates were incubated at $35 \circ \mathrm{C}$ aerobically for $18 \mathrm{~h}$. After the incubation period, the diameters of the zones of inhibition were measured using a ruler. The experiments were replicated three times for each microorganism.

The overall procedures experimented in the study of $\mathrm{BC}$ from fermented sweet glutinous rice (FSR) compared with the control coconut and the profile of culturing conditions could display in Fig. 1.



Fig. 1. The procedures in the study involving $\mathrm{BC}$ production by FSR with A. xylinum and Acetobacter spp. compared with that made by coconut juice. 


\section{Results}

\subsection{Culturing Conditions of BC produced by FSR}

BC produced from the culture of FSR was similar to that of fermented coconut juice. It had brown color and at least 5-15 mm thickness (Fig. 2) but more tuff was detected (data not shown).



Fig. 2. Bacterial cellulose $(\mathrm{BC})$ membrane obtained by fermented sweet glutinous rice (FSR), vinegar, with A. xylinum TISTR 080, static condition at $30^{\circ} \mathrm{C}$ for 5 days.

In Fermented Sweet Rice sample (FSR), optimum temperature and $\mathrm{pH}$ profiles of $\mathrm{BC}$ and $A$. xylinum yield were at $25-30^{\circ} \mathrm{C}$ and $\mathrm{pH} 4-7$, respectively. In Figure $3 \mathrm{~A}$, the highest $\mathrm{BC}$ yield at approximately $12-13 \mathrm{~g} / \mathrm{L}$ could be detected and, the highest growth of the bacteria required the $\mathrm{pH}$ of $4-4.5$. Figure $3 \mathrm{~B}$ indicated that the bacteria disliked the media having lower $\mathrm{pH}$ than 4 or higher than 7. The $\mathrm{pH}$ in the range of 4-7 was the optimum growth indicating the suitable $\mathrm{pH}$ for $\mathrm{BC}$ production (Fig. 3).

(A) Temperature Profile

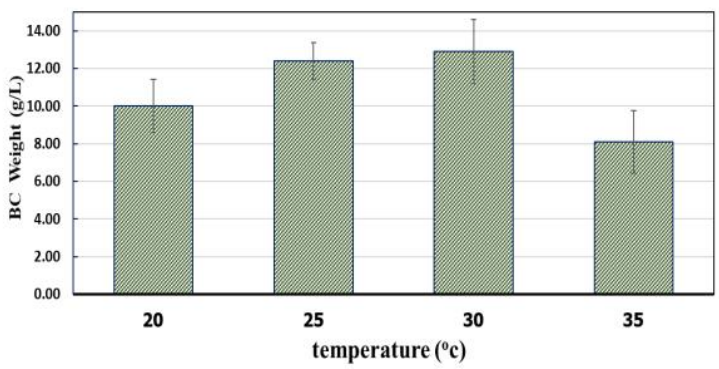

(B)

pH profile

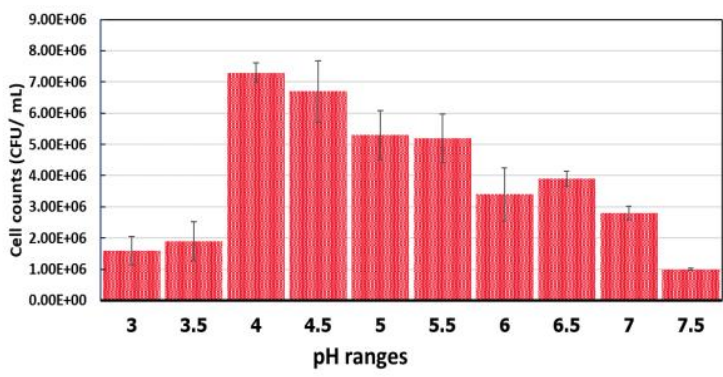

Fig. 3. Temperature (A) and $\mathrm{pH}(\mathrm{B})$ affecting on the fermentation process of fermented sweet glutinous rice (FSR) for BC production.
Carbon sources were required for the growth of the bacteria (Acetobacter spp.). In the study, after cultivation for 15 days, the culture added with glucose provided the highest $\mathrm{BC}$ production $(12 \mathrm{~g} / \mathrm{L})$, whereas molasse and fructose produced less $\mathrm{BC}$ (10 and $5 \mathrm{~g} / \mathrm{L}$, respectively) (shown in Fig. 4A). More addition of carbon sources (20-25 $\mathrm{g} / \mathrm{L}$ ) affected more thickness of BC membrane (5-10 mm), while very thin $\mathrm{BC}$ with the thickness less than $2 \mathrm{~mm}$ was seen in the broth without carbon source supplement (data not shown). The shorten incubation period to obtain the maximum BC yield was found in the media supplemented with glucose $(15 \mathrm{~g} / \mathrm{L}$ within 4 days, while lower $\mathrm{BC}$ yield was detected in the medium having molasse $(10 \mathrm{~g} / \mathrm{L}$ within 15 day). Additionally, the least $\mathrm{BC}$ yield was in that having fructose ( $5 \mathrm{~g} / \mathrm{L}$ within 7 days) (Fig. 4B). It seemed molasse could enhance more $\mathrm{BC}$ yield than fructose, but the bacteria feasibly took longer time in metabolizing molasse having complexed sugar moieties.

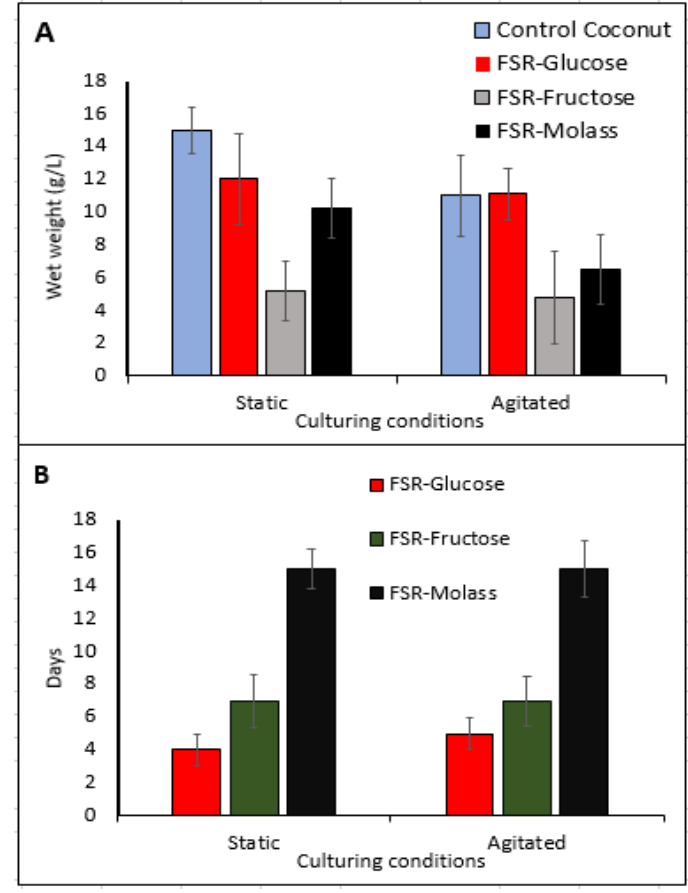

Fig. 4. BC production depending on the supplemented carbon sources including glucose, fructose, and molasse in FSR after cultivation for 3 days (A) and maximum incubation period required to obtain highest $\mathrm{BC}$ yields from SFR (B). Mixed diverse Acetobacter species promoting $\mathrm{BC}$ production.

When comparing the ability in $\mathrm{BC}$ production between the pure species of Acetobacter xylinum TISTR 086 and mixed cultures of Acetobacter spp., the mixed Acetobacter cultures could produce higher amount of BC within 5 days, whereas only one Acetobacter species required at least 9 days to provide the maximum $\mathrm{BC}$ yield (approximately $15 \mathrm{~g} / \mathrm{L}$ from both samples) (Fig. 5A and 5B). However, slightly more total cell counts of pure culture were detected in the HS agar than those of mixed cultures $\left(1.0 \times 10^{7} \mathrm{CFU} / \mathrm{mL}\right.$ within 5 days from pure culture and $7.06 \times 10^{6} \mathrm{CFU} / \mathrm{mL}$ within 5 days from mixed cultures) (Fig. 5C and 5D). It was possible that the pure culture could grow faster and possibly expressed less 
competition action than mixed cultures. Similarly, acetic producing bacterial species could have high competition and thus were not be able to enhance the $\mathrm{BC}$ yield, while the various Acetobacter species could interact each other and enhance more BC yield.

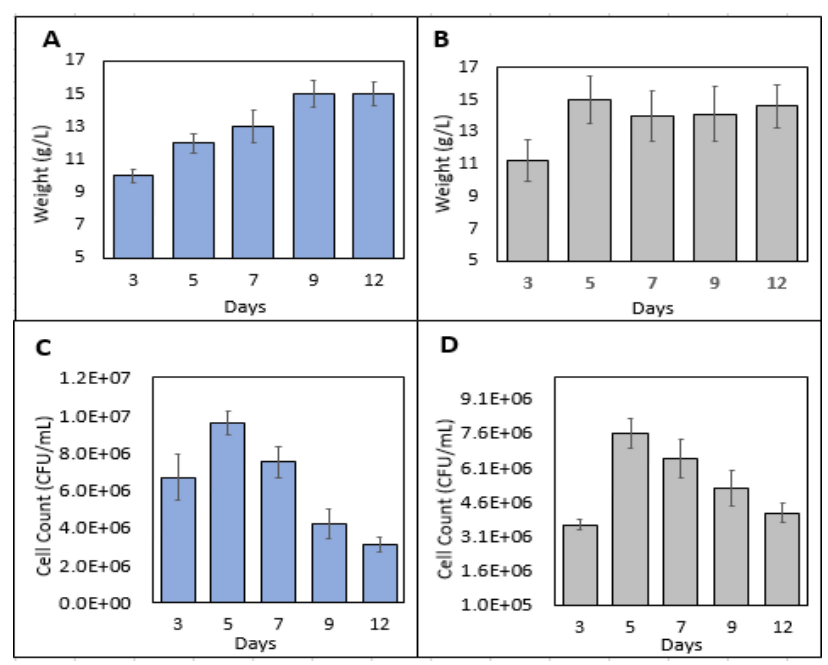

Fig. 5. The comparison of Acetobacter species affecting the fermentation process of FSR. BC yield (A, B) and the cell growth $(\mathrm{C}, \mathrm{D})$ were obtained from the cultures of Acetobacter xylinum (A, C) and mixed Acetobacter spp (B, D), respectively.

\subsection{The Importance of Carbon Source on BC Yield}

The addition of glucose as carbon source affected BC production, not for the growth of the bacteria. In Figure 6 , high $\mathrm{BC}$ production replenished with additional carbon source (glucose at $20 \mathrm{~g} / \mathrm{L}$, but possible reduction to $5 \mathrm{~g} / \mathrm{L}$, data not shown) was detected as shown high BC contents (12 g/L in FSR sample) compared to that in coconut sample (16 g/L) (Figure 6A and 6B). No glucose addition in both FSR (an example of FSR in Fig. 6A) and coconut samples could maintain high bacterial cells within 5-7 days $\left(6.5-7.5 \times 10^{6} \mathrm{CFU} / \mathrm{mL}\right)$ but the cells produced very low BC yields (3.5 and $4.6 \mathrm{~g} / \mathrm{L}$ for FSR and coconut sample, respectively (Fig. 6B). It was possible that carbon source remained during fermentation seemed to be used up for the bacterial growth and not enough for the enzymatic activity involving in BC synthesis [19] (Fig. $6 \mathrm{~A}$ and $6 \mathrm{~B})$.

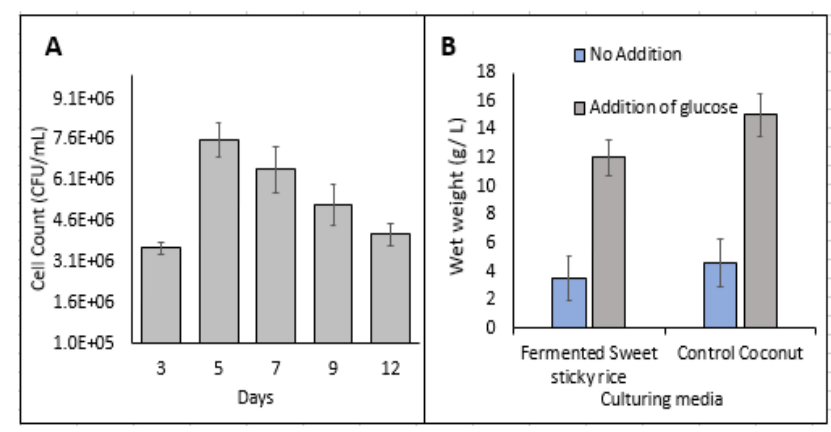

Fig. 6. The effects of carbon source to mixed Acetobacter cultures involving in $\mathrm{BC}$ production. The cell counts of FSR without glucose (A) and the BC yields with and without glucose (B).

\subsection{Antibacterial activity of Ag-BC product}

The data in Fig. 7B indicated that the $\mathrm{BC}$ impregnated with AgNPs at the concentration of at least 50 ppm could demonstrate antibacterial activity by producing $6 \mathrm{~mm}$ clear zone on the plate within $24 \mathrm{~h}$. The disk diffusion method was sensitive enough to indicate high biocide activity of AgNP at $10 \mathrm{ppm}$ presented on the surface and inside of BC obtained from FSR, comparable to that of coconut juice treated sample (Fig. 7A) and similar to another report that applied AgNPs into $\mathrm{BC}$ made from fermented coconut juice [13]. In anti-wound pathogen, when using AgNP with the concentration of $100 \mathrm{ppm}$, the species $E$. coli was the most susceptible, E. coli was more sensitive to AgNPs than $S$. aureus and $P$. aeruginosa as its bigger clear zone (shown in Fig. 7C; 25,17 , and $15 \mathrm{~mm}$ for $E$. coli, P. aeruginosa, and $S$. aureus, respectively. At lower concentration of $50 \mathrm{ppm}$, $S$. aureus and $P$. aeruginosa showed insignificant differences for the inhibition zone (the diameter of 6.4 and $6.2 \mathrm{~mm}$ ). It was possible that the AgNPs at low concentration needed time for contacting and diminishing the bacteria and it would be better action of Ag-gel/liquid phase [20]. However, the AgNP impregnation method presented in the same manner of other several nano- and biocompatible polymeric materials, such as hepatite, collagen, aloe vela, alginate, and gelatin [21-26]. The membrane with small pore sizes occured both on the BC surface and inside the membrane helped to trap the AgNPs. More appropriate moisture content of Agmodified dressing material facilitates the wound-healing process and prevents colonization of infectious bacteria as effective bacteriocidal activity on the wound pathogen. The impart effects for $\mathrm{Ag}$ precipitate in pores on the surface of Ag-BC samples could possible affect the lower bacteriocidal activity [27], shown at Ag concentration higher than $100 \mathrm{ppm}$ and thus indifferent inhibition zones at 25 and $27 \mathrm{~mm}$ diameter detected in $\mathrm{Ag}-\mathrm{BC}$ at 100 and 1,000 ppm (Fig. 7C and 7D).

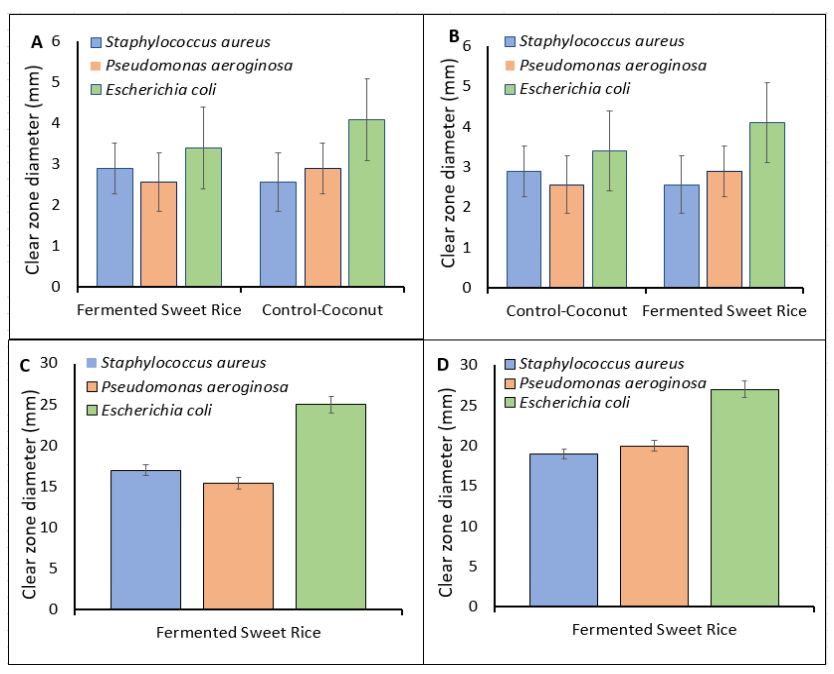

Fig. 7. Antibacterial activity of Ag-BC produced from FSR after impregnated with AgNPs at concentration of $10 \mathrm{ppm}$ (A) $50 \mathrm{ppm}$ (B) $100 \mathrm{ppm}$ (C) and 1,000 (D). The control of fermented coconut juice shown in (A) was also soaked with AgNPs at the concentration of $10 \mathrm{ppm}$. 


\section{Discussions}

The approaches of the development of BC produced by the vinegar fermentation of sweet glutinous rice, could be another good start, not only fermented coconut juice, in production of bacterial cellulose that can be developed to be new nanomaterials related biomedical with biodegradable and harmless qualification. This knowledge related to $\mathrm{Ag}-\mathrm{BC}$ can further tentatively inspire and expand its potential application of other new biomaterial fields. Not only wound dressing by blending AgNPs with gelling agent [28], the hydrated BC membrane with nanofibers can be used to produced nanopore matrixes and big surface with stronger and more toughness than the other gel forms. BC from both FSR and coconut should be another good medium choice for accommodating high availability of AgNPs solution, especially enough diffusion and strengthen inhibition effects onto the wound dressing pathogens.

Major advantageous option of Ag- BC from FSR, using sweet glutinous rice, was cheaper and more convenient protocol in fermentation process than that made by coconut sample since the fermentation could be done without the needs of ethanol supplementation and tentative less needs of carbon sources [29]. The obtained $\mathrm{BC}$ had light weight than common $\mathrm{BC}$ produced by coconut juice (data not shown). However, the physio-mechanical, and biochemical characterization, and biodegradability should also be analysed to extend the possible to use FSR made BC.

The variation of $\mathrm{BC}$ is depended on its structure and properties and could be adjusted by the bacterial strain selection, amount of carbon sources as well as constant incubation temperature [30]. The properties of BC including mechanical, porosity, fibre length and its diameter are additionally varying according to the strains, compositions of the culturing media, fermentation condition (static and agitated culturing) and post processing (purification) [31, 32]. After these factors were successfully optimized, BC membrane from FSR with acceptable mechanical properties including compactness, uniform distribution and increased toughness will be ready for the future large-scale application, especially in vivo applications for further medical treatment and possible temporary skin substitute or wound dressing.

\section{Conclusion}

The approach of bacterial cellulose production by the fermentation of sweet glutinous rice is one good choice of a natural renewable and biodegradable polymer. Its nanofiber surfaces can expand its potential application in the biomedical fields. In this study, the optimized conditions including diversified species, temperature, types of carbon sources, incubation conditions, and duration of $\mathrm{BC}$ production can be a first step to indicate its comparable ability to common $\mathrm{BC}$ type made by coconut for application in biomedical field, especially antagonism onto the pathogen.
This research work was financially supported by Rajamangala University of Technology Phra Nakhon, Thailand.

\section{References}

1. H. El-Saied, A.H Basta, R.H. Gobran, PolymerPlastics Tech. Eng. 43, 797-820 (2004)

2. Z. Shi, Y. Zhang, G.O. Phillips, G. Yang, Food Hydrocoll. 35, 539-545 (2014)

3. F. Esa, S.M. Tasirin, N.A. Rahman, Agri Sci Proc. 2, 113 - 119 (2014)

4. O. M. Alvarez, M. Patel, J. Booker, L. Markowitz, Wounds 16, 224-233 (2014)

5. M. Hubbe, A. Ferrer, P. Tyagi, O.J. Rojas, A Review Biores. 12(1), 2143-2233 (2017)

6. S. M. Mohammad, N.A. Rahman, Adv Biol Res. 8(6), 307-313 (2014)

7. L. M. M. Costa, G.M. Olyveira, P. Basmaji, L.X. Filho, J Bio Tissue Eng. 2, 185-196 (2012)

8. S. Quintavalla, L. Vicini, Meat Sci. 62(3), 373-380 (2002)

9. D.A. Pereira de Abreu, J.M. Cruz, L.P. Paseiro, Food Rev Inter. 28(2), 146-187. (2011).

10. J.D. Fontana, A.M. De Souza, C.K. Fontana, I.L. Torriani, J.C. Moreschi, B.J. Gallotti, S.J. De Souza, G.P. Narcisco, J.A. Bicharaand, L.F.X. Farah, Appl Biochem Biotech. 24-25, 253-64 (2019)

11. W. Czaja, D. Romanovicz, J.R.M. Brown, Cellulose 11, 403-411 (2004)

12. D. Klemm, D. Schumann, U. Udhardt, S. Marsch, Prog Poly Sci. 26, 1561-1603 (2001)

13. H.S. Barud, T. Regiani, R.F.C. Marques, W.R. Lustri, Y. Messaddeq, S.J.L. Ribeiro, J Nanomat. 721631, 1-8 (2011)

14. M.M. Abeer, A.M.C. Mohd, C. Martin, J Pharm Pharm. 66(8), 1047-1061 (2014)

15. K. Novotna, P. Havelka, T. Sopuch, K. Kolarova, V. Vosmanska, L. Bacakova, Cellulose 20, 2263-2278 (2013)

16. D.A. Schumann, A. Wippermann, J. Klemm, D.O. Kramer, F. Koth, D. Kosmehl, H. Wahlers, T.S. Salehi-Gelani, Cellulose 16(5), 877-885 (2009)

17. M. Kishida, Y. Seike, H. Kawasaki, Biocontrol Sci. 14(3), 119-122 (2009)

18. Y.S. Hwan, O.S. Lee, I.S. Lee, H.S. Kim, T.S. Yu, Y.J. Jeong, J Microbiol Biotech. 14(2), 276-283 (2004)

19. M.W. Ullah, M.U. Islam, S. Khan, Y. Kima, J.K. Parka, Carbohydr Poly. 136, 908-916 (2015)

20. A. Nakayama, A. Kakugo, J.P. Gong, Y. Osada, M. Takai, T. Erata, S. Kawano, Functional Mat. 14, 1124-1128 (2004)

21. K.A. Zimmermann, J.M. Leblanc, K.T. Sheets, R.W. Fox, P. Gatenholm, Materials Sci Eng C. 31, 43-49 (2011)

22. Z. J. Cai, G. Yang, J Appl Poly Sci. 1205, 2938-2944 (2011)

23. O. A. Saibuatong, M. Phisalaphong, Carbohydrate Poly. 79, 455-460 (2010) 
24. N. Chiaoprakobkij, N. Sanchavanakit, K. Subbalekha, P., Pavasant, M. Phisalaphong, Carbohydrate Poly. 853, 548-553 (2011)

25. S. B., Lin, C. P., Hsu, L. C., Chen, H. H. Chen, Food Hydrocoll. 23, 2195-2203 (2009)

26. K. A. Zimmermann, J. M. Leblanc, K. T. Sheets, R. W. Fox, P. Gatenholm, Mat Sci Eng C. 31, 43-49 (2011)

27. Y. Dahman, J. Nanosci. Nanotech. 9, 5105-5122 (2009)
28. A., Nakayama, A. Kakugo, J.P. Gong, Y. Osada, M Takai, T. Erata, S. Kawano, Advance Functional Mat. 14, 1124-1128 (2004)

29. M., Kishida, Y. Seike, H. Kawasaki, Biocontrol Sci. 14(3), 119-122 (2009)

30. S. Sheykhnazari, T. Tabarsa, A. Ashori, A. Shakeri, M. Golalipour, Carbohydrate Poly. 86, 1187-1191 (2011)

31. A. Sani, Y. Dahman, J Chem Tech Biotech. 85(2), 151-164. (2010)

32. W. Tang, S. Jia, Y. Jia, H. Yang, World J Microbiol Biotech. 26(1), 125-131. (2009) 